\title{
Effect of the Directional Characteristics of the Microphone Pair MD441 on the Measurement of the Speech Transmission Index STIPA and Comprehensibility CIS
}

\author{
René Drtina $^{1, a}$, Pavel Fejfar ${ }^{1, b}$, Josef Sedivy ${ }^{1, c}$, Oldrich Turecek ${ }^{1, d}$ \\ 1,2,3,4 University of Hradec Kralove, Faculty of education, Department of technical subject \\ Rokitanskeho 62, 50003 Hradec Kralove, Czech Republic \\ arene.drtina@uhk.cz, bpavel.fejfar@uhk.cz, cjosef.sedivy@uhk.cz, doldrich turecek@uhk.cz
}

Keywords: speech transmission index, clarity, sensor, directional characteristic, base sensors, pressure microphone, gradient microphone, hyperkardiod microphone.

Abstract: Acoustic information transmission path even today maintains a dominant position. Concept clarity measurement method Stipe is based on the empirical finding that variation the level of speech signals carry important information relating to speech intelligibility. Modulation of speech arises from acoustic separation of words, sentences and phonemes, which are the basic elements of speech.

\section{Introduction}

The outputs of the existing analyzes suggest that the measurement of the speech transmission index STIPA (although it is a standard set objective method) can not agree with logatomic tests for evaluating the transmission properties of conference areas. Especially in language classrooms, then we can assume a fatal impact classroom acoustics and the properties of the transmission channel on the quality of teaching. The aim of the specific research project SV 2113 Faculty of Education in 2015, called "The dependence of the measured speech transmission index STIPA directional and frequency characteristics of acoustic receivers." The research project builds on previous projects that followed the speech intelligibility in conference and lecture halls, and compared the possibility of measuring methods, whose output is a single number parameter. This parameter should ideally be the responsibility of logatomic recognizability, as determined by standardized logatomic tests [1]. We proceed from the fact that a normal healthy individual hearing is binaural and that, therefore, the measurement should respect human directional hearing. Our aim is to propose a modification of the measuring system so that the correlation of results of signal measurements and tests were logatomic greatest, ideally to the correlation coefficient $\rho$ CIS LOG as possible to approximate to value 1.

\section{Twin sensor MD441}

For the simulation of directional hearing in the acoustic space classrooms and lecture we used a pair of Sennheiser of hypercardiod sensors MD441U.

Directional characteristics of sensors can be described so. Directivity factor $\mathrm{Q}_{\mathrm{p}}$, which according to [5] applies

$$
\mathrm{Q}_{\mathrm{p}}=\frac{U_{0}^{2}}{\frac{1}{4 \pi} \iint U_{\psi}^{2} \mathrm{~d} \Omega}
$$

where $U_{0}$ is the output voltage of the sensor plane waves along the acoustic axis of the transducer. Denominator represents the output voltage of the sensor in a perfectly diffuse field. Directivity factor but not determinative of the geometry of the directional characteristics.

Ideal hypercardiod sensor can create a pair of point-gradient of sensors zero and first order [2]. Typically utilizes a combination of pressure and speed sensors whose output voltages are added together. The output voltage of the speed sensor. Common mode voltage pair is then 
$U=p \eta_{2}+p \eta_{1} \cos \alpha=p \eta_{2}\left(1+\frac{\eta_{1}}{\eta_{2}} \cos \alpha\right)$

where $p$ is the acoustic pressure, $\eta 1$ and $\eta 2$ are sensitive sensors and $\alpha$ is a departure from the acoustic axis of the sensor. For ratio $\eta 1 / \eta 2=0.75$ directivity factor reaches a maximum value of $\mathrm{Qp}=4$. Direction function point hypercardiod sensors so we can ideally describe a standardized formula

$$
A=0,25+0,75 \cos \alpha
$$

where $A$ is the normalized output level of the sensor $(A \max =1)$. Theoretic ideal directional function of the sensor is ideal hypercardiod in Fig.1.

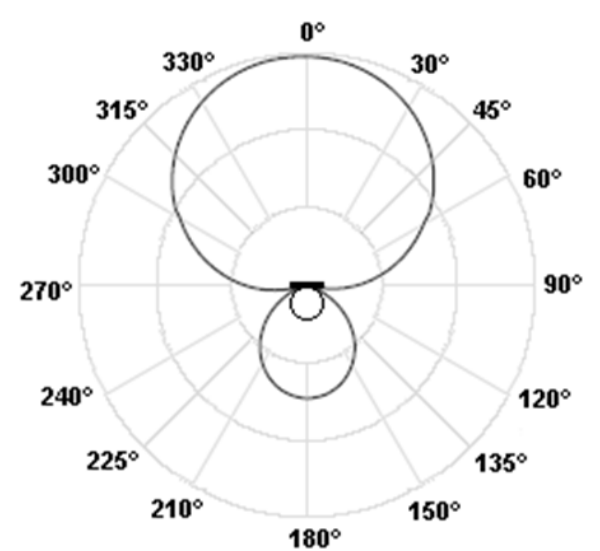

Fig. 1 Theoretic functions hypercardioid directional sensor

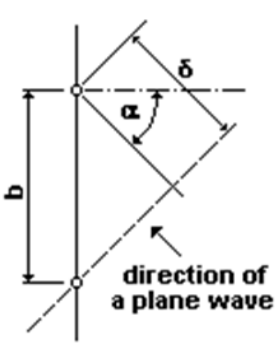

Fig. 2 Deriving a common-mode voltage sensing twin

If we use a pair of identical sensors on remote base $b$, lands plane wave whose propagation direction makes with the acoustic axis of the sensor angle $\alpha$, a second sensor is delayed $\Delta t$, which corresponds path difference $\delta$ (Fig. 2). For common-mode voltage level and thus the directional characteristics of two identical point hypercardiod sensors base b we get, using equation (3)

$$
A=(0,25+0,75 \cos \alpha)\left(1+\cos 2 \pi f \frac{b}{c} \sin \alpha\right)
$$

Depending based sensors B according to equation (4) changes the resultant directional characteristic of the sensor pair. Figure 3 is a theoretical courses directional scanning functionality of identical twin point hypercardiod sensors base $b=6 \mathrm{~cm}$ and $\mathrm{b}=17 \mathrm{~cm}$. At relatively small base $(6 \mathrm{~cm})$ leads to narrowing of the directional characteristics with minimal side lobes. With the increase in the base is greatly narrow directional characteristic for lower frequencies, but for higher frequency leads to the formation of side lobes and the directional characteristic is cleaved into narrow strips [3].

For the sensors real situation is much more complicated. Above all, a realistic final dimensions of the sensor, it is not a point. Hypercardiod microphone membrane generally has the shape of a spherical cap and around its own acoustic sensors are different circuits. On Fig. 4 and Fig. 5 are measured by the directional characteristics of the transducer pair Sennheiser MD441U base $b=$ $6 \mathrm{~cm}$ and $\mathrm{b}=17 \mathrm{~cm}$. Measurement of directional characteristics we realized multitone signal in an anechoic chamber with a reference microphone Brüel \& Kjær analyzer and BK Pulse 3560C. The measurement results confirm the theoretical distinction directional characteristics of a pair of spot. Sensor directional characteristics of real sensors with finite dimensions and the accuracy of the model calculations in accordance direction of the side lobes [4]. 

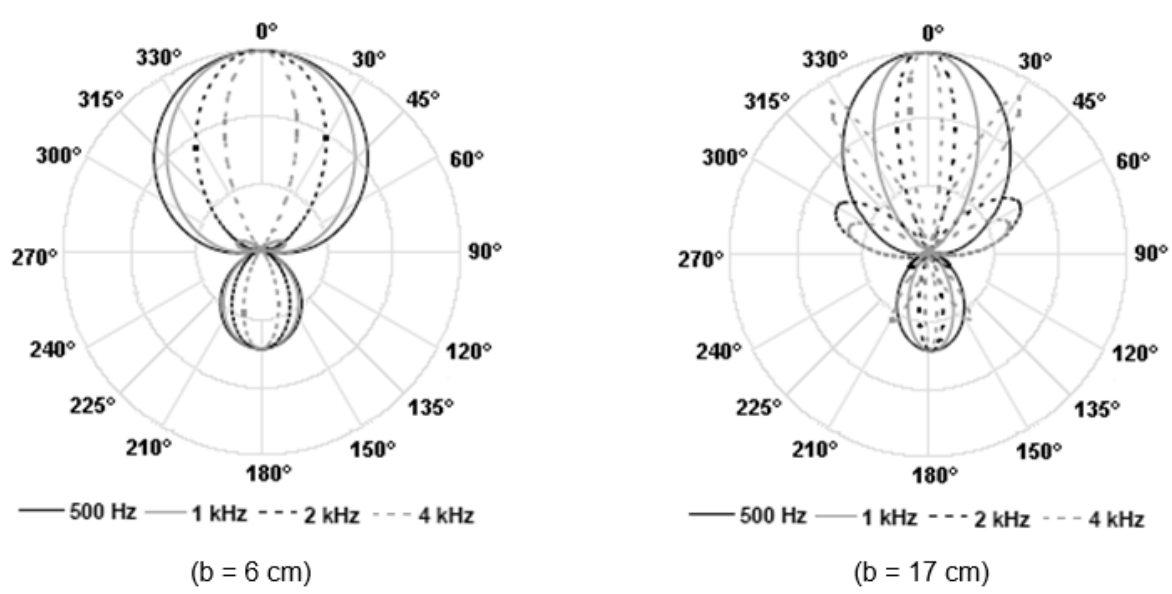

Fig 3. Theoretical courses feature a pair of directional hypercardioid sensors
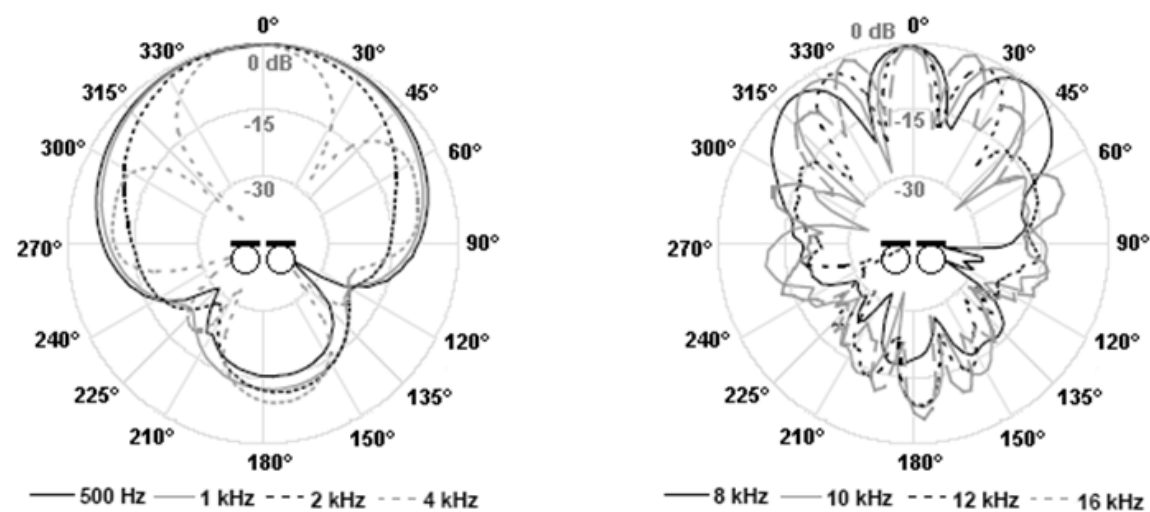

Fig.4 directional characteristics of sensor pair MD441U $(b=6 \mathrm{~cm})$
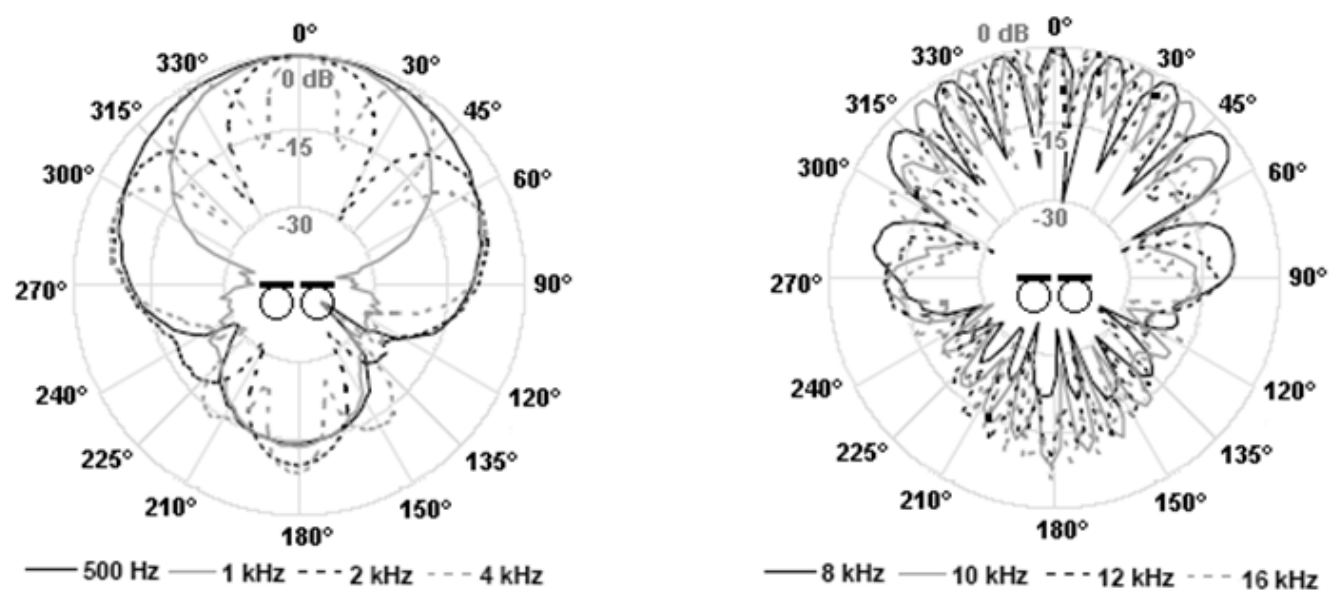

Fig.5 Directional characteristics of sensor pair MD441U $(b=17 \mathrm{~cm})$

\section{Results of a comparative index measurements speech transmission}

Effect of directional characteristics of sensors for measuring the index of speech transmission STIPA and clarity CIS we tested in space with a reverberation time of $1,5 \mathrm{~s}$ at a distance of 1-6 m from the reference emitter NTi TalkBox. On Fig. 6, the values measured Stipe and CIS scanning twin compared with the values measured by pressure microphone NTi 2201 with "balls" characteristic. While pressure microphone largely represents unilaterally deaf man, the directional characteristics of the sensor pair is approaching binaural hearing mono signal. 

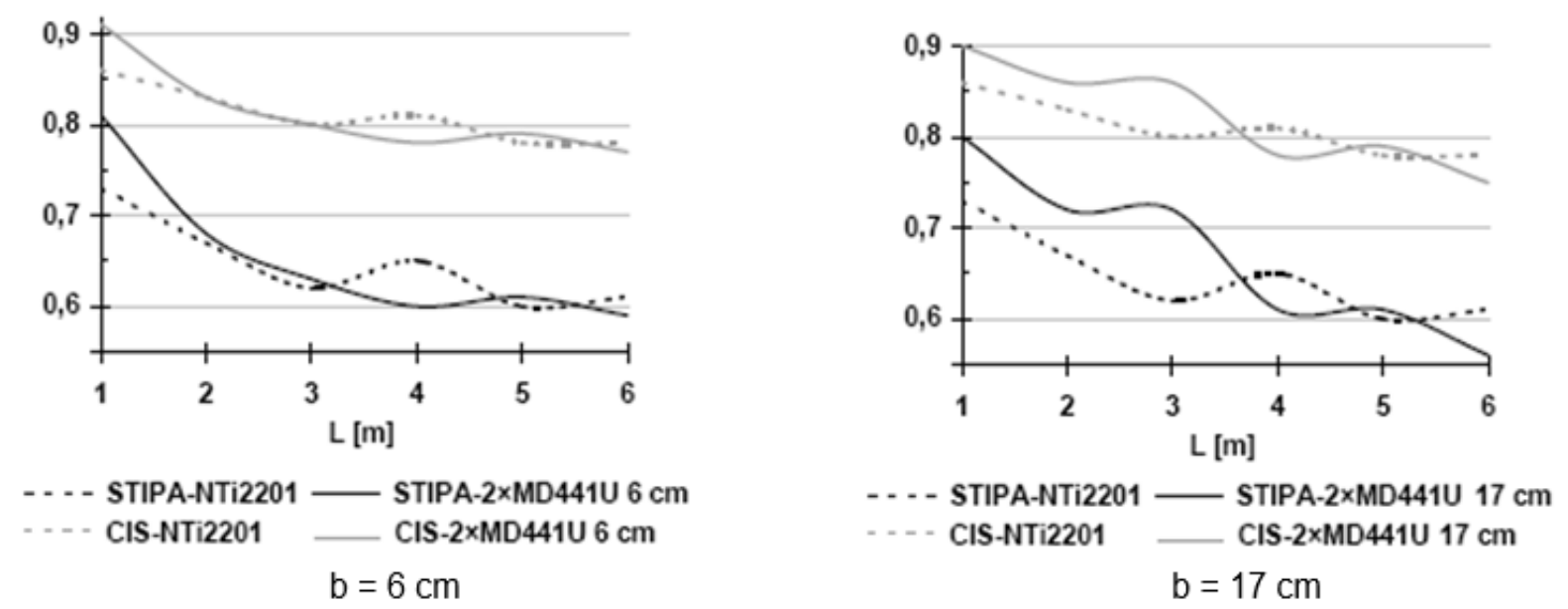

Fig.6 Comparison of measured values STIPA and CIS

\section{Conclusion}

The results of the measurements, we can infer that the directional characteristics of the sensor pair has a significant influence on the measurement results, especially in the free field and a pair of matching pitch with bases ears. In the diffuse field results are comparable.

Partial results of research have shown that the influence of directional characteristics of the used sensors to the measured value speech transmission index STIPA and derived values of clarity, the CIS is significant. Another direction of research activities, therefore, will lead to finding appropriate directional characteristics and validation of the measured values with the results of the tests logatomic so that one-numeric measurement method is comparably sensitive to syllable intelligibility, especially in the range from 85 to $100 \%$, where the standard moving test results logatomic in confernce and lecture halls, where the most stringent requirements set loss to $5 \%$ readability.

\section{Acknowledgements}

The article was prepared with the support of specific research project SV 2133 PdF UHK dependence of the measured speech transmission index STIPA directional and frequency characteristics of acoustic receivers.

\section{References}

[1] F. W. Yuan, Q. Deng, L. H. Zhao, "Radiation Resistance Experiments of Image Sensor and Acoustic Sensor", In: Advanced Materials Research, Vols. 591-593, pp. 1571-1574, Nov. 2012.

[2] S. Liu, Y. Lan, Q. Li, "Design of Underwater Acoustic Vector Sensor and its Elastic Suspension Element", In: Applied Mechanics and Materials, Vols. 713-715, pp. 569-572, Jan. 2015.

[3] G D. Y. Huang, "A New Method to Measure the Absorption Coefficient Based on the Sound Pressure Delaying", In: Advanced Materials Research, Vols. 834-836, pp. 1156-1160, Oct. 2013.

[4] Y. Zhang, B.B. Shen, S.J. Meng, "Research of HRTF Character of Head-Mounted Microphone Array", In: Applied Mechanics and Materials, Vol. 743, pp. 479-483, Mar. 2015 